Supporting Information

\title{
Shape-Tunable $\mathrm{SrTiO}_{3}$ Crystals Revealing Facet-Dependent Optical and
}

\section{Photocatalytic Properties}

Pei-LunHsieh, ${ }^{\dagger}$ GollapallyNaresh, $\stackrel{\ddagger}{\S}$ Yu-Sheng Huang, ${ }^{\dagger}$ Chun-Wen Tsao, ${ }^{\|}$Yung-Jung Hsu, Lih-JuannChen, ${ }^{*}, \dagger, \perp$ Michael H. Huang $*,+, \perp$

†Department of Materials Science and Engineering, National Tsing Hua University, Hsinchu 30013, Taiwan

\#Department of Chemistry, National Tsing Hua University, Hsinchu 30013, Taiwan $\S$ Department of Chemical and Biological Engineering, University of Sheffield, United Kingdom

\#Department of Materials Science and Engineering, National Chiao Tung University, Hsinchu 30010, Taiwan

${ }^{\perp}$ Frontier Research Center on Fundamental and Applied Sciences of Matters, National Tsing Hua University, Hsinchu 30013, Taiwan

Table S1. Reagent amounts used for the synthesis of $\mathrm{SrTiO}_{3}$ cubes with tunable edge lengths.

\begin{tabular}{|c|c|c|c|c|c|c|c|c|}
\hline \multicolumn{2}{|c|}{ Cubes } & $\mathrm{TiCl}_{4}$ & Water & Ethanol & $\mathrm{SrCl}_{2} \cdot 6 \mathrm{H}_{2} \mathrm{O}$ & LiOH & Temperature & Time \\
\hline & $\sim 290 \mathrm{~nm}$ & $0.026 \mathrm{~mL}$ & $1 \mathrm{~mL}$ & $1.5 \mathrm{~mL}$ & $0.070 \mathrm{~g}$ & $3.7 \mathrm{~mL}(3 \mathrm{M})$ & $70{ }^{\circ} \mathrm{C}$ & $3 \mathrm{~h}$ \\
\hline & $\sim 250 \mathrm{~nm}$ & $0.026 \mathrm{~mL}$ & $0.5 \mathrm{~mL}$ & $2 \mathrm{~mL}$ & $0.070 \mathrm{~g}$ & $3.7 \mathrm{~mL}(3 \mathrm{M})$ & $70^{\circ} \mathrm{C}$ & $3 \mathrm{~h}$ \\
\hline & $\sim 200 \mathrm{~nm}$ & $0.026 \mathrm{~mL}$ & $0 \mathrm{~mL}$ & $2.5 \mathrm{~mL}$ & $0.070 \mathrm{~g}$ & $3.7 \mathrm{~mL}(3 \mathrm{M})$ & $70^{\circ} \mathrm{C}$ & $3 \mathrm{~h}$ \\
\hline & $\sim 160 \mathrm{~nm}$ & $0.031 \mathrm{~mL}$ & $0 \mathrm{~mL}$ & $2.5 \mathrm{~mL}$ & $0.084 \mathrm{~g}$ & $3.7 \mathrm{~mL}(3 \mathrm{M})$ & $70^{\circ} \mathrm{C}$ & $3 \mathrm{~h}$ \\
\hline
\end{tabular}


Table S2. Reagent amounts used for the preparation of $164 \mathrm{~nm} \mathrm{SrTiO}{ }_{3}$ cubes, $181 \mathrm{~nm}$ edge-truncated cubes, and $168 \mathrm{~nm}\{100\}$-truncated rhombic dodecahedra.

\begin{tabular}{|c|c|c|c|c|c|c|c|}
\hline \multirow[b]{2}{*}{ Cubes } & $\mathrm{TiCl}_{4}$ & Water & Ethanol & $\mathrm{SrCl}_{2} \cdot 6 \mathrm{H}_{2} \mathrm{O}$ & LiOH & Temperature & Time \\
\hline & $0.031 \mathrm{~mL}$ & $0 \mathrm{~mL}$ & $2.5 \mathrm{~mL}$ & $0.084 \mathrm{~g}$ & $3.7 \mathrm{~mL}(3 \mathrm{M})$ & $70^{\circ} \mathrm{C}$ & $3 \mathrm{~h}$ \\
\hline \multirow{2}{*}{$\begin{array}{c}\{110\} \text {-truncated } \\
\text { cubes }\end{array}$} & $\mathrm{TiCl}_{4}$ & Water & Hexanol & $\mathrm{SrCl}_{2} \cdot 6 \mathrm{H}_{2} \mathrm{O}$ & LiOH & Temperature & Time \\
\hline & $0.208 \mathrm{~mL}$ & $2 \mathrm{~mL}$ & $0.85 \mathrm{~mL}$ & $0.560 \mathrm{~g}$ & $3.7 \mathrm{~mL}(3 \mathrm{M})$ & $200^{\circ} \mathrm{C}$ & $20 \mathrm{~h}$ \\
\hline \multirow[b]{2}{*}{$\begin{array}{c}\{100\} \text {-truncated } \\
\text { rhombic } \\
\text { dodecahedra }\end{array}$} & $\mathrm{TiCl}_{4}$ & Water & Ethylene Glycol & $\mathrm{SrCl}_{2} \cdot 6 \mathrm{H}_{2} \mathrm{O}$ & LiOH & Temperature & Time \\
\hline & $0.026 \mathrm{~mL}$ & $2 \mathrm{~mL}$ & $0.85 \mathrm{~mL}$ & $0.070 \mathrm{~g}$ & $3.7 \mathrm{~mL}(3 \mathrm{M})$ & $200^{\circ} \mathrm{C}$ & $20 \mathrm{~h}$ \\
\hline
\end{tabular}

Table S3. Surface area and volume calculations for the determination of relative amounts of particles needed possessing the same total particle surface area.

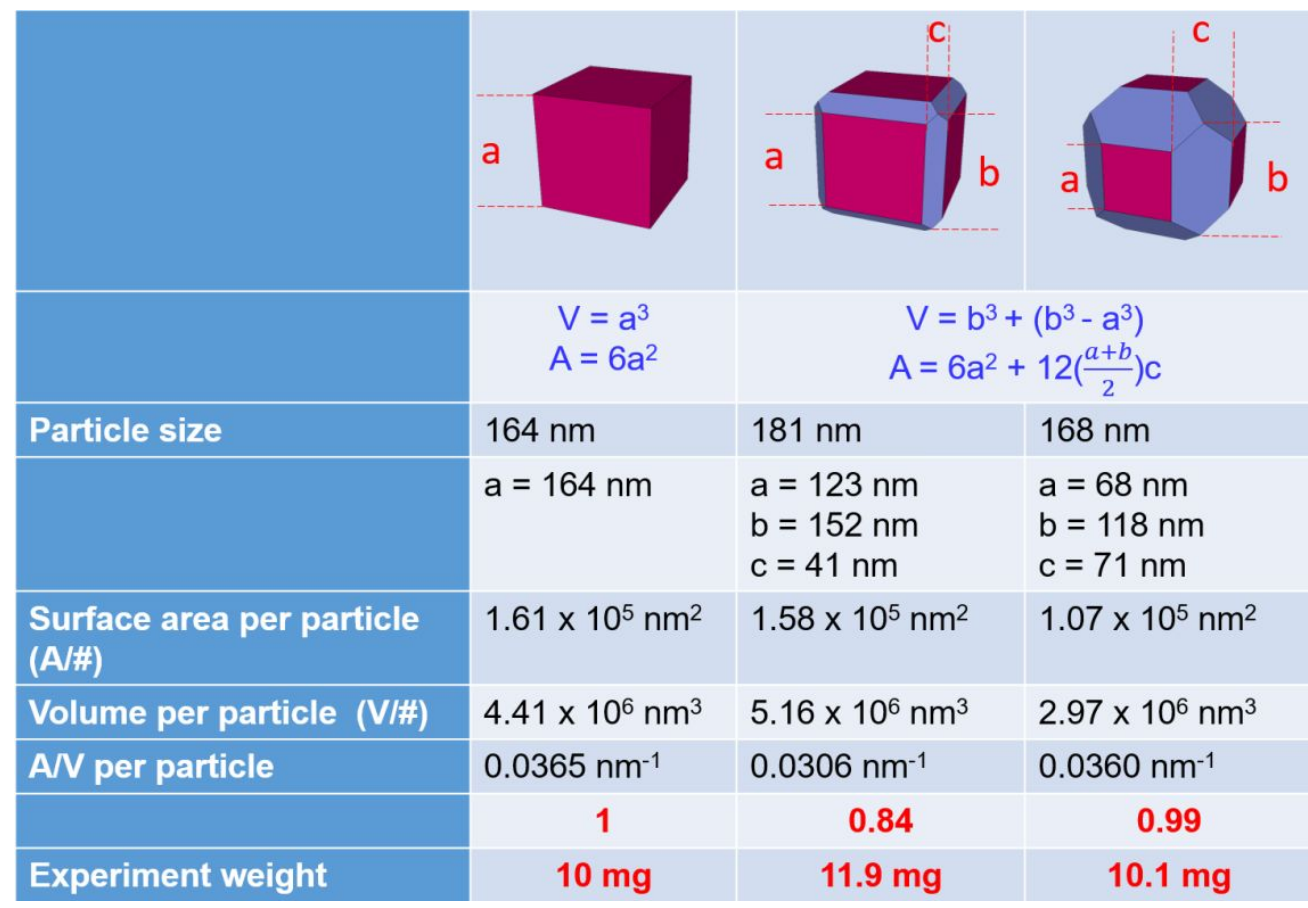




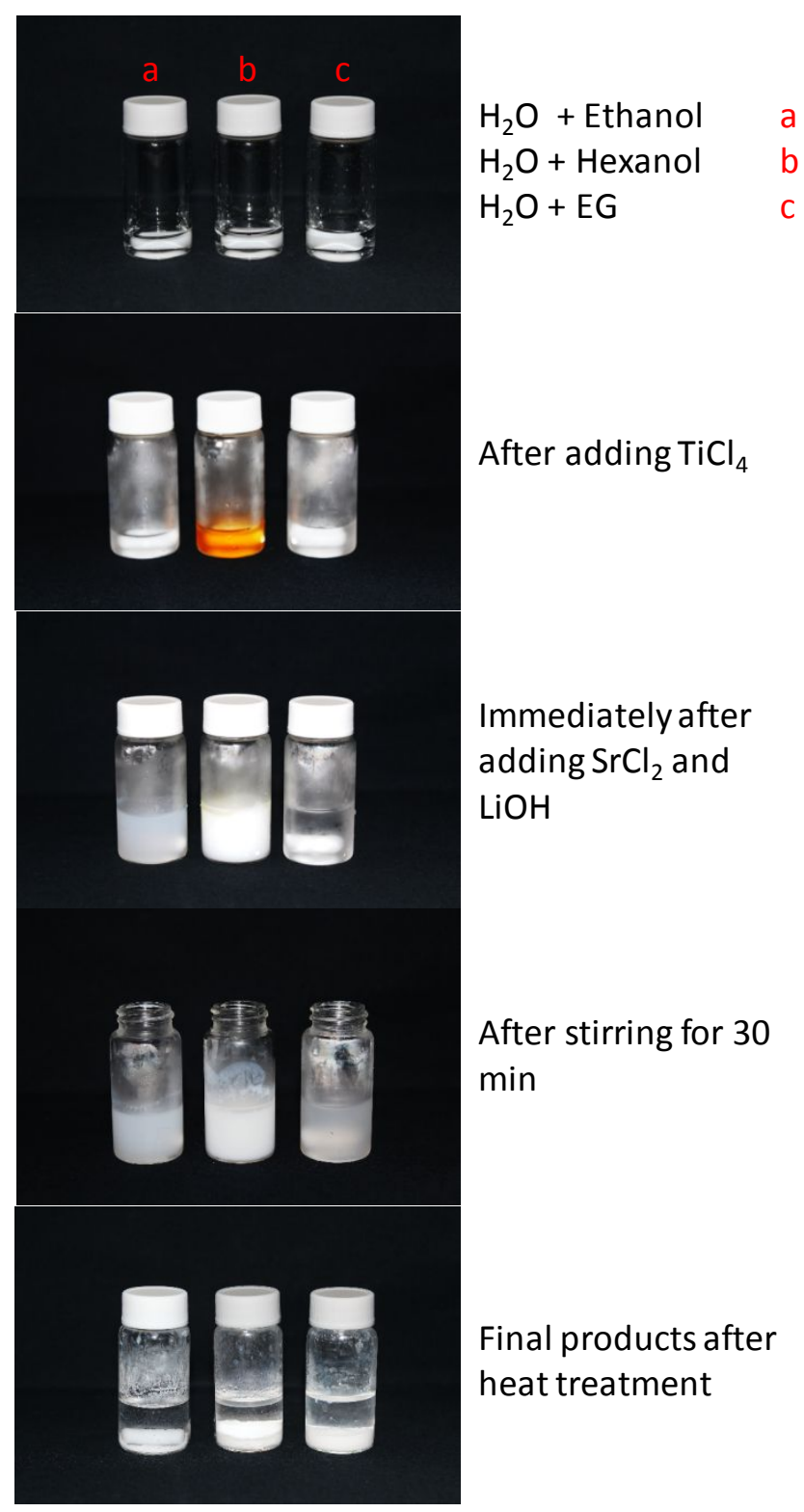

Figure S1. Photographs taken at various time points in the synthesis of $\mathrm{SrTiO}_{3}$ (a) cubes, (b) edge-truncated cubes, and (c) $\{100\}$-truncated rhombic dodecahedra. Addition of $\mathrm{TiCl}_{4}$ to hexanol makes the solution orange in color. The color remains after adding $\mathrm{SrCl}_{2}$, but becomes white after the introduction of $\mathrm{LiOH}$.

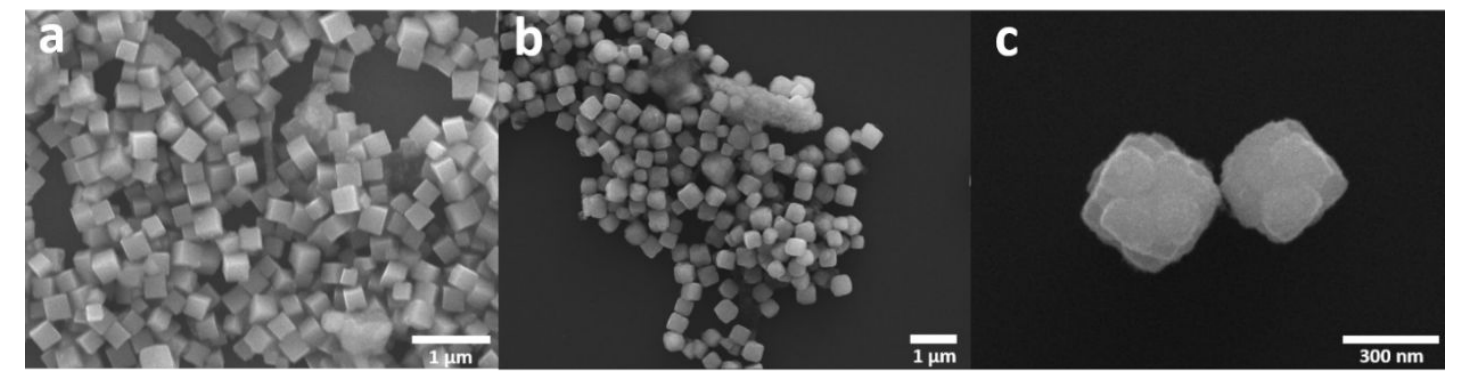

Figure S2. SEM images of $\mathrm{SrTiO}_{3}$ cubes synthesized in the presence of (a) $\mathrm{LiOH}$, (b) $\mathrm{NaOH}$, and (c) $\mathrm{KOH}$. 


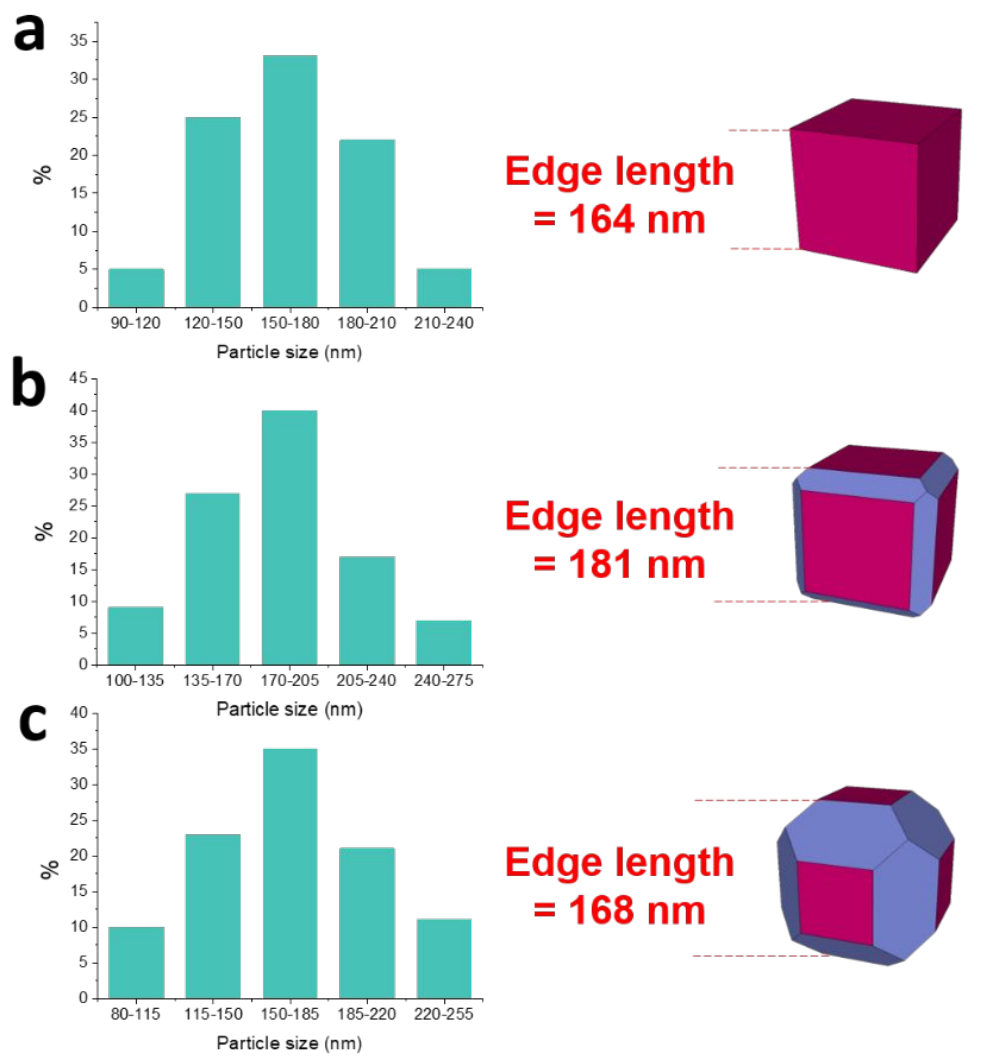

Figure S3. Particle size distribution histograms of the synthesized $\mathrm{SrTiO}_{3}$ (a) cubes, (b) edge-truncated cubes, and (c) $\{100\}$-truncated rhombic dodecahedra.

Table S4. Exact XRD peak positions for the three shapes of prepared $\mathrm{SrTiO}_{3}$ crystals.

\begin{tabular}{|c|c|c|c|c|c|c|c|c|c|}
\hline \multirow{2}{*}{$\mathrm{SrTiO}_{3}$} & \multicolumn{9}{|c|}{2 Theta } \\
\hline & (100) & (110) & (111) & $(200)$ & (210) & (211) & (220) & (300) & (310) \\
\hline Cubes & 22.55 & 32.09 & 39.59 & 46.08 & 51.84 & 57.25 & 67.20 & 71.93 & 76.41 \\
\hline $\begin{array}{c}\{110\} \text {-truncated } \\
\text { cubes }\end{array}$ & 22.72 & 32.36 & 39.91 & 46.42 & 52.28 & 57.70 & 67.76 & - & 77.10 \\
\hline $\begin{array}{c}\{100\} \text {-truncated } \\
\text { rhombic } \\
\text { dodecahedra }\end{array}$ & 22.73 & 32.34 & 39.97 & 46.43 & 52.29 & 58.06 & 67.77 & - & 77.13 \\
\hline
\end{tabular}



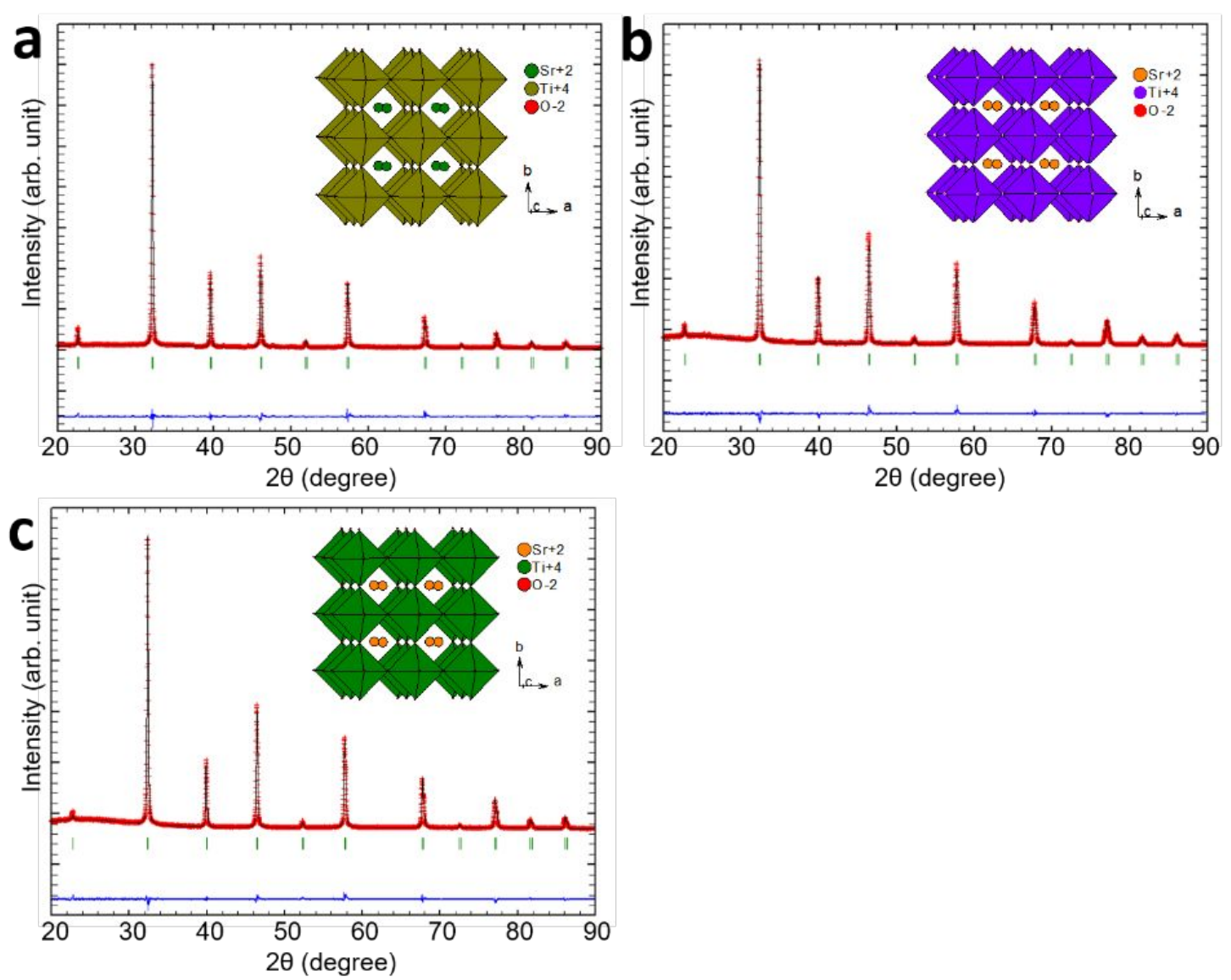

Figure S4. Rietveld refinement of the $\mathrm{SrTiO}_{3}$ (a) cubes,(b) edge-truncated cubes and (c) $\{100\}$-truncated rhombic dodecahedra. Observed (+), calculated (-) and difference (bottom) profiles are shown. The vertical bars represent the Bragg positions.

Table S5. Selected bond lengths $(\AA)$ of the obtained $\mathrm{SrTiO}_{3}$ samples.

\begin{tabular}{|c|c|c|c|c|c|c|}
\hline \multirow{2}{*}{$\mathrm{SrTiO}_{3}$} & \multirow{2}{*}{$\begin{array}{c}\mathbf{a}=\mathbf{b}=\mathbf{c} \\
(\AA)\end{array}$} & \multicolumn{5}{|c|}{ Bond Length $(\AA)$} \\
\hline & & $\mathrm{Ti}-\mathrm{Ti}$ & Ti-O & $\mathrm{Ti}-\mathrm{Sr}$ & $\mathrm{Sr}-\mathrm{Sr}$ & $\mathrm{Sr}-\mathrm{O}$ \\
\hline Cubes & 3.936 & 3.936 & 1.968 & 3.409 & 3.936 & 2.783 \\
\hline $\begin{array}{c}\{110\} \text {-truncated } \\
\text { cubes }\end{array}$ & 3.912 & 3.912 & 1.956 & 3.388 & 3.912 & 2.766 \\
\hline $\begin{array}{c}\{100\} \text {-truncated } \\
\text { rhombic } \\
\text { dodecahedra }\end{array}$ & 3.909 & 3.909 & 1.955 & 3.385 & 3.909 & 2.764 \\
\hline
\end{tabular}



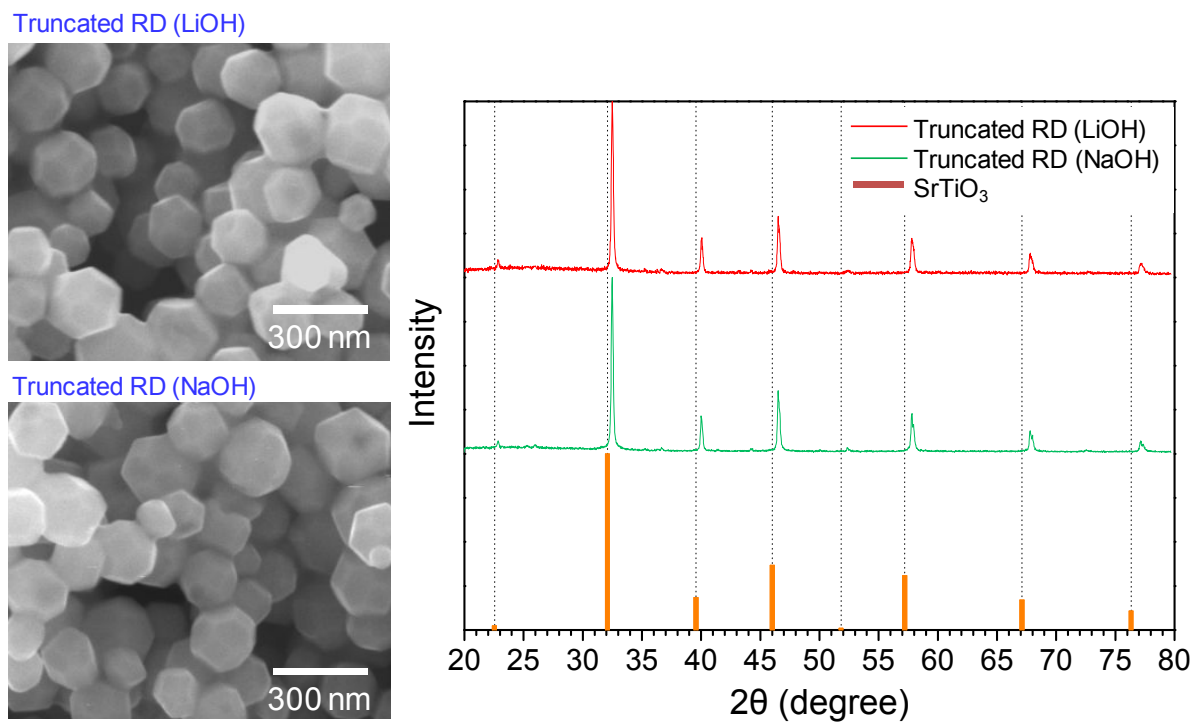

Figure S5. SEM images of the $\mathrm{SrTiO}_{3}$ truncated rhombic dodecahedra synthesized with the addition of $\mathrm{LiOH}$ or $\mathrm{NaOH}$ and their XRD patterns. 

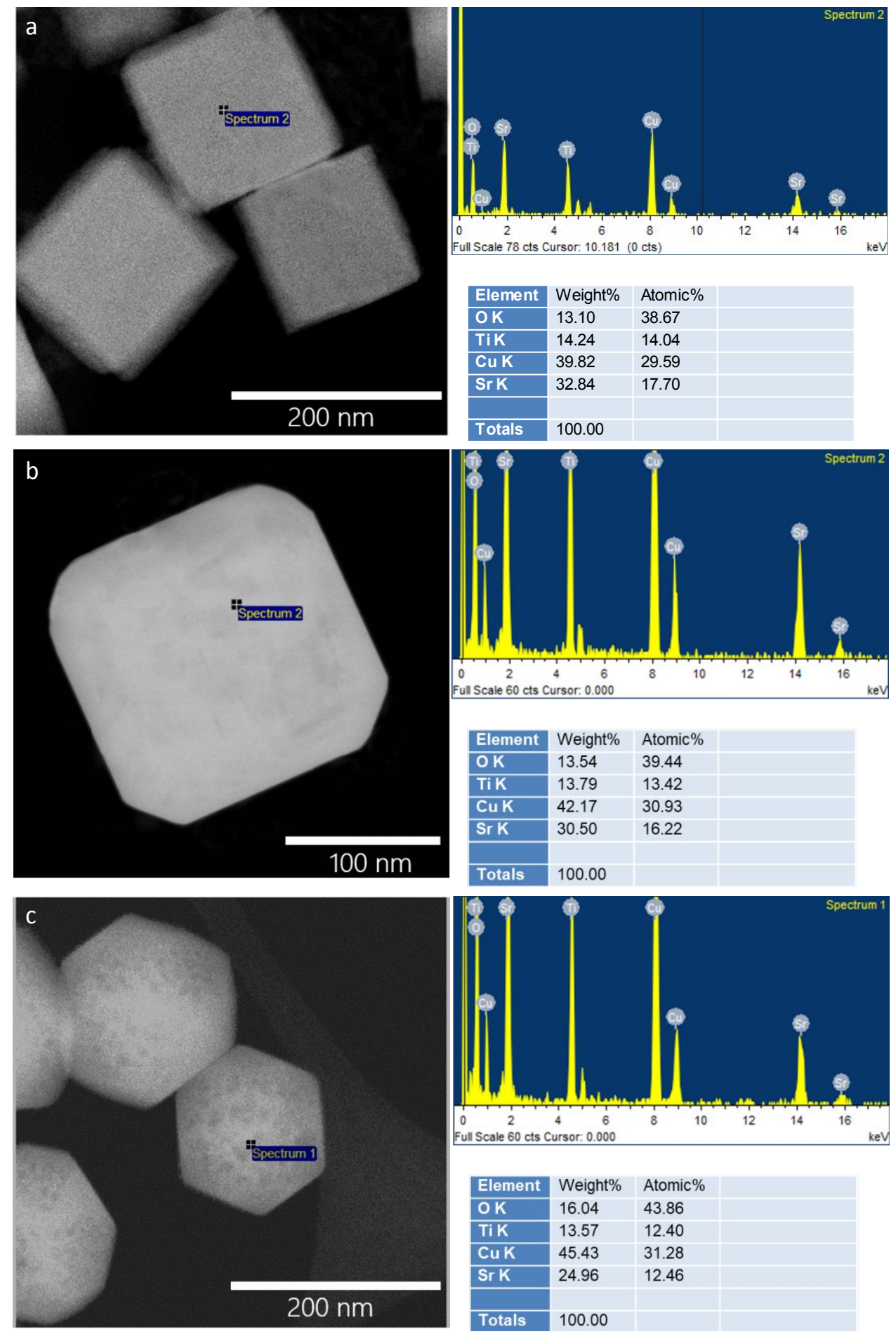

Figure S6. EDS analysis on a single $\mathrm{SrTiO}_{3}$ (a) cube, (b) edge-truncated cube, and (c) truncated rhombic dodecahedron. Atomic ratios of Sr:Ti:O are roughly 1:1:3 for these samples. 

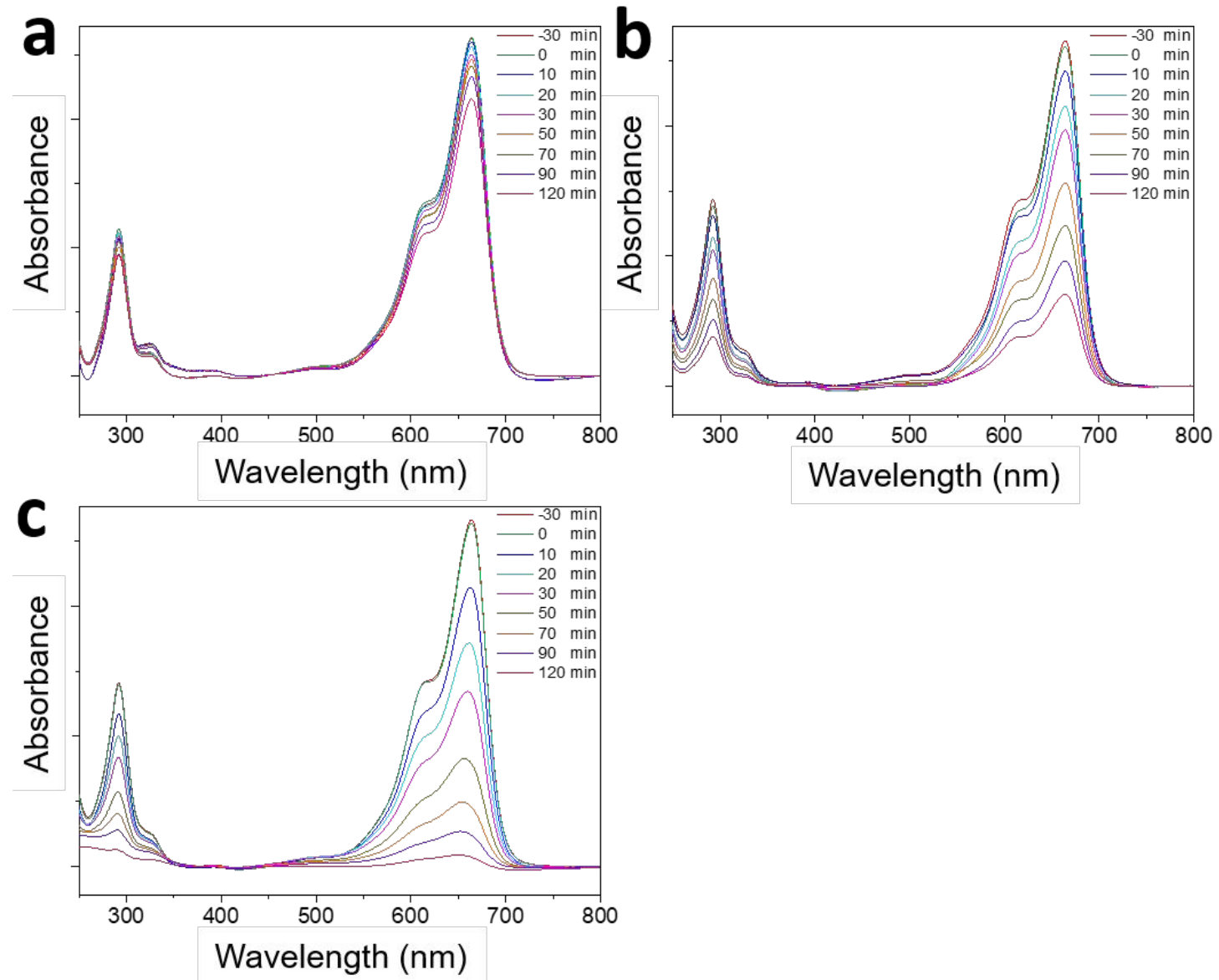

Figure S7. UV-vis absorption spectra of methylene blue as a function of the photodegradation time using $\mathrm{SrTiO}_{3}$ (a) cubes, (b) edge-truncated cubes, and (c) $\{100\}$-truncated rhombic dodecahedra as the photocatalysts. Light irradiation begins at time zero.

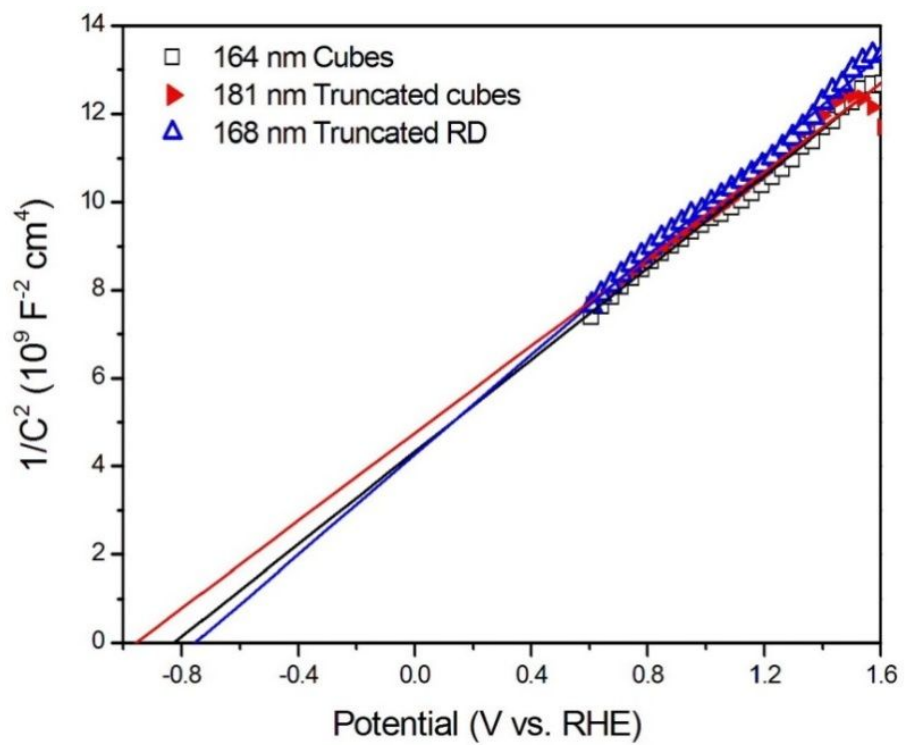

Figure S8. Mott-Schottky plots recorded on the three different $\mathrm{SrTiO}_{3}$ crystals. The straight lines represent the linear fits of the data. 


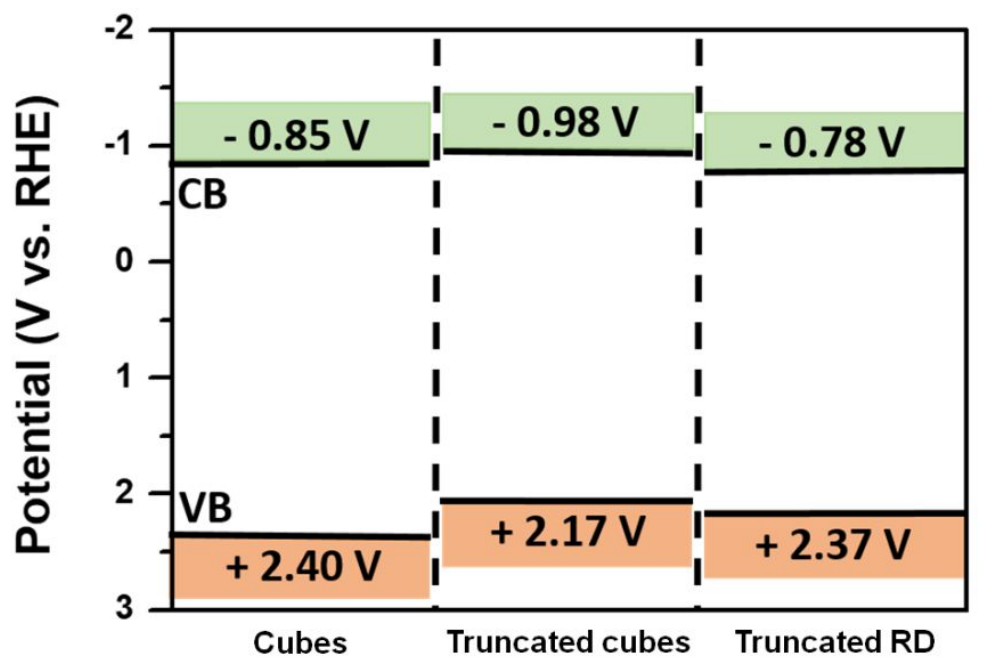

Figure S9. Band structures for the three different $\mathrm{SrTiO}_{3}$ crystals derived from the Mott-Schottky plots. 